\title{
Three-Dimensional Modeling of Human Placental Terminal Villi
}

\author{
Romina Plitman Mayo ${ }^{\mathrm{a}, \mathrm{b}}$, D. Stephen Charnock-Jones ${ }^{\mathrm{a}, \mathrm{c}}$, Graham J. Burton ${ }^{\mathrm{a}}$, Michelle L. \\ Oyen $^{\mathrm{a}, \mathrm{b}}$ \\ ${ }^{a}$ Centre for Trophoblast Research, Department of Physiology, Development and Neuroscience, University of \\ Cambridge, Cambridge, UK \\ ${ }^{b}$ Nanoscience Centre, Department of Engineering, University of Cambridge, Cambridge, UK \\ ${ }^{c}$ Department of Obstetrics $\&$ Gynaecology, University of Cambridge, Cambridge, UK
}

\begin{abstract}
Introduction: Placental transport is the main factor affecting the health and development of the fetus. Due to the placenta's geometrical and mathematical complexity, the structurefunction relations of placental terminal villi have not been successfully modeled. Hence, a novel modeling approach is proposed.

Methods: Computational models of four different specimens were generated from the three dimensional reconstruction of confocal laser scanning microscopic image stacks. To evaluate the capabilities of the proposed methodology, stationary oxygen diffusion transport was calculated in the terminal villus volumes.

Results: The reconstructions automatically provided the spatial arrangement of the fetal capillaries inside the terminal villi. The surface and volume ratios between the fetal capillaries and the villus were also calculated, and the effects of model parameters on the placental diffusive capacity were assessed by parametric analysis.

Discussion: The potential of three-dimensional reconstructions combined with finite element analysis as a research tool for the human placenta was tested. The methodology herein could serve in the future as a simulation platform for complicated in vivo and in vitro scenarios.
\end{abstract}

Keywords: Oxygen Transport, Placenta, Terminal Villi, Confocal Laser Scanning Microscopy, Diffusion Analysis

\section{Introduction}

Placental oxygen transport is believed to be the most important function of the human placenta. At the smallest branches of placental villous trees, diffusive transport takes place due to concentration gradients that arise between the outer surface of the trophoblast layer and the inner surface of the fetal capillary endothelium [1]. These terminal villi are believed to be the most important sites for gas exchange due to their thin barriers between the maternal and fetal bloodstreams [2]. Therefore, their structure-function relations are of great interest to placental physiologists.

Histological methods have been very popular for the assessment of terminal villous geometry since they provide an approximation of the complex three-dimensional (3D) 
structure [3-5]. However, the published data are sparse and inconsistent, mainly due to the different investigation and fixation procedures employed. The 3D architecture of the villus was first visualized and quantified using scanning electron microscopy and vascular corrosion casts, $[6,7]$ which allowed for easier and more exact approaches than histology. Currently, 3D imaging techniques such as X-ray microcomputed tomography (microCT) and confocal laser scanning microscopy (CLSM) are providing new methods for studying vessel alterations in humans and animals. The 3D fetoplacental vascular bed of normal and pathological human placentae has been investigated using microCT $[8,9]$; however, its resolution is not enough for the acquisition of a detailed structure of the terminal villi. CLSM has provided unique information regarding the 3D spatial arrangement of microvascular beds in normal and pathological placentae, during the first [10] and third [11, 12] trimesters. Volumetric reconstructions of CLSM images could provide useful insights if combined with mathematical modeling.

Mathematical models of placental exchange function were initially described more than 40 years ago $[2,13]$. The different modeling approaches can be classified into two broad groups: based on experimentally obtained geometry and on parametrized geometry. The first group is mostly composed by studies that adapted the two-dimensional (2D) morphometric model developed for the gas exchange in the lungs [14] to the placenta $[3,5,15-18]$. A different methodology was employed by Gill et al. [2], who used digital photomicrographs to extract the $2 \mathrm{D}$ geometry of the villi and their respective capillaries. The extent of diffusional screening in placental villi was then numerically calculated. Their work is pioneering and has the advantage that it can be performed in multiple villi simultaneously. Similarly, Chernyavsky et al. [19] estimated the distribution of villous branches in the intervillous space, and solute transport was modeled. However, when experimental data are not available, such as for the branching villus trees, the only way of solving a more comprehensive mathematical model is by simplifying the geometry to known geometrical shapes - $e$. g. tubes and ovals. These type of studies form the second and smallest group of placental modeling [20-22]. Thus, most of the studies performed on the oxygen transport in the human placenta have successfully modeled it in $1 \mathrm{D}$ and $2 \mathrm{D}$, but those who attempted to do so in $3 \mathrm{D}$ were required to simplify the geometrical structure. For a detailed review on models of oxygen transport in the placenta the reader is referred to Serov et al. [23].

In the current study, three-dimensional reconstructions of terminal villi and their capillary beds are combined with finite element (FE) analysis to test their potential as an investigative tool. The technique is tested by solving stationary oxygen diffusive transport. This approach accounts for specimen-specific geometry where the contribution of the villous membrane and fetal capillary variability is directly incorporated, while their effect in 3D is quantified and visualized. It also provides the opportunity to investigate in detail the structure-function relation in the terminal villi. 


\section{Material and Methods}

\subsection{Specimen Preparation}

A fresh healthy placenta delivered by Cesarean section at term was obtained at the Department of Obstetrics \& Gynaecology in Addenbrooke's Hospital for perfusion fixation [24], with ethical permission and informed written consent. Two undamaged and clot-free lobules suitable for perfusion were identified and the amnion was removed from the chorionic plate above these. The chorionic artery supplying each lobule was cannulated and the draining vein was cut to allow perfusate flow. Fetal blood was cleared from the lobules with phosphate buffered saline (PBS). The lobules were then fixed by perfusion with $10 \%$ formalin (20 min) followed by removal with PBS (an additional $20 \mathrm{~min}$ ). The vessels were perfused with PBS containing green fluorescein isothiocyanate (FITC) conjugated Ulex lectin (FL-1061, Vector Laboratories Peterborough, Cambridgeshire, UK) that has an excitation and emission maximum wavelength of $495 \mathrm{~nm}$ and $515 \mathrm{~nm}$ respectively. Once the vessels were filled, the solution was left in place for $30 \mathrm{~min}$ and then removed by flushing with PBS. The two lobules were processed at 2 different perfusion pressures: $100 \mathrm{mmHg}$ (specimen 1 and 2) and $30 \mathrm{mmHg}$ (specimen 3 and 4) representing the extremes of the physiological pressures [25]. Intermediate villi with small clumps of terminal villi attached were dissected with needles and incubated for 10 minutes in DiI (D-282, Life technologies, CA, USA) to stain the villous membranes (excitation maxima wavelength of $549 \mathrm{~nm}$ and emission maxima wavelength of $565 \mathrm{~nm}$ ).

The specimens were scanned using a Leica SP2 CLSM (Leica Microsystems, Wetzlar, Germany) with an x25, 0.95NA objective lens. Based on the quality of the images (which is a function of the quality of the perfusion), four terminal villi were selected for quantitative analysis. For each sample, image stacks with a resolution of $0.277 \mu \mathrm{m}^{2} \mathrm{x} 2 \mu \mathrm{m}$ were automatically generated.

\subsection{Geometry Reconstruction}

The CLSM data were imported into 3DSlicer [26], an open-source software for image analysis. The stacks were converted to grayscale images and the geometries of interest were automatically detected by the thresholding method, where each grayscale image was transformed into a binary one [27]. Thereafter, manual cleaning based on human interpretation was needed to remove image artifacts and define unclear boundaries; for example, when two close but obviously different capillary segments appear merged within the same boundary they are manually disconnected and the resulting gap equals the pixel size $(0.277$ $\mu \mathrm{m})$. For better processing, a Gaussian blur smoothing algorithm [27] was applied to further clean and reduce noise. Due to the lack of information regarding the material properties (diffusion coefficients) of the trophoblast epithelium, basement membrane, connective tissues and capillary endothelium, they were modeled together as a single entity called the villous membrane. Three-dimensional triangulated meshes were generated from the segmented stacks with the help of an interpolation algorithm [28] and converted into point clouds (a collection of points that describes the surface of a given shape) by employing an in-house code that extracts the triangle vertices. The solid bodies (volumetric geometry) which mimic 


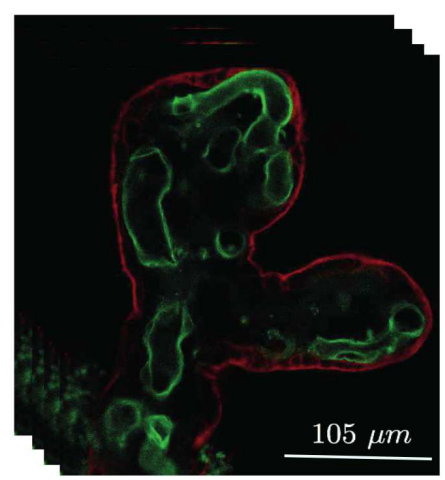

(a)

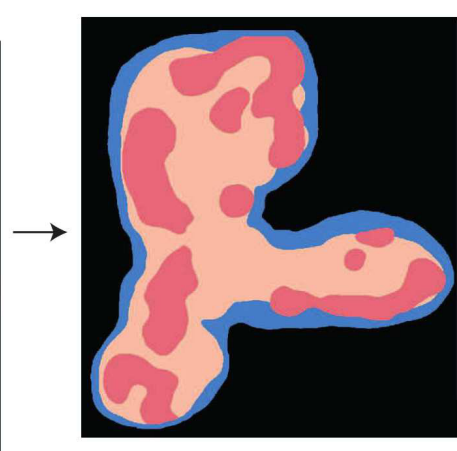

(b)

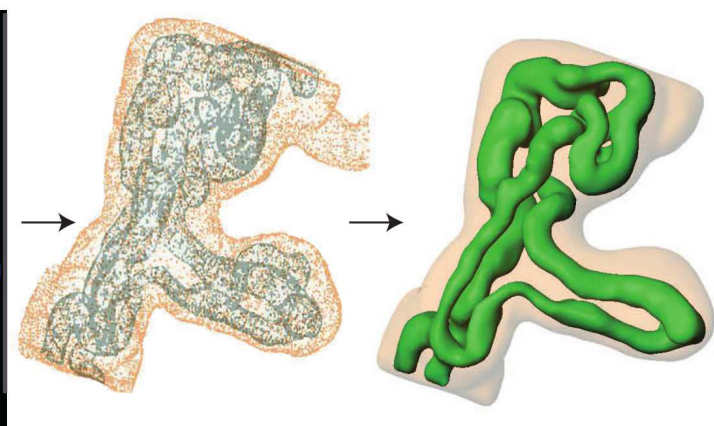

(c) (d)

Figure 1: Schematic flow chart for the reconstruction of 3D geometries from CLSM stacks. (a) Typical 2D CLSM slice where the trophoblastic layer (red) and the fetal capillaries (green) are visible, (b) Typical 2D label-map with colors for the different tissues: blue for the trophoblastic membrane, beige for the stroma and pink for the fetal capillaries, (c) Point cloud of the villi and fetal capillary surfaces and (d) 3D Solid bodies.

the specimen specific terminal villi and fetal capillaries were automatically generated in SolidWorks-2013 software (SolidWorks Corporation, MA, USA) by employing the PointCloud function. The overall 3D reconstruction process is schematically shown in Figure 1.

The microscopic size of the samples challenges the verification of the geometries. Therefore, a methodology that enables the estimation of the reconstruction accuracy was developed. Four $r=90 \mu \mathrm{m}$ and three $r=4 \mu \mathrm{m}$ spheres were scanned with equal parameters as the villous specimens, but with 3 different slice thicknesses. The spheres were reconstructed and analyzed. The data indicated that the precision of the geometrical reconstruction mostly depends on the slice thickness. For the given geometries, a $1 \%$ error is expected in each direction $(x, y, z)$.

\subsection{Computational Model}

The 3D solid bodies were imported and implemented using the commercial FE solver COMSOL Multiphysics 5.2 (COMSOL, Inc., Burlington, MA, USA). The villous membrane and fetal capillaries were treated as different domains to allow for different boundary conditions. These two domains were meshed separately with continuity across the boundary, by the COMSOL automesher. Due to the volume and complexity of each specimen, the size of the meshes differed greatly ranging from 430,000 to 825,000 tetrahedral elements. A typical mesh sensitivity procedure [29] in which the meshes are refined and the convergence of the results is checked was performed. Minimum element size was less than minimum thickness to reduce numerical errors.

A passive oxygen transport problem was considered to evaluate the capabilities of the presented method. The oxygen is assumed to diffuse through the villous membrane following Fick's second law of diffusion, Eq. 1, which states that the oxygen volume flow (with time $t$ ) 
across a membrane is related to its physical dimensions $(x, y, z)$, the driving concentration gradient, $\nabla \mathbf{C}$, and the diffusion coefficient, $D$.

$$
\frac{\partial \mathbf{C}}{\partial t}=D\left(\frac{\partial^{2} \mathbf{C}}{\partial x^{2}}+\frac{\partial^{2} \mathbf{C}}{\partial y^{2}}+\frac{\partial^{2} \mathbf{C}}{\partial z^{2}}\right)=D \nabla^{2} \mathbf{C}
$$

The oxygen concentration at the villous membrane corresponds to the maternal blood concentration, which varies according to the position of the terminal villus in the intervillous space and the proximity to other terminal villi. However, since both parameters are unknown a constant oxygen concentration surrounding the villi $\left(\mathrm{C}_{\text {villi }}=1 \cdot 10^{-9} \mathrm{~mol} / \mathrm{m}^{3}\right)$ was assumed. Since it is not possible to determine from 2D CLSM images which capillaries carry oxygenated blood and which carry deoxygenated blood, all fetal capillaries were treated as carrying arterial deoxygenated blood and thus acting as perfect sinks for oxygen $\left(\mathrm{C}_{\text {capillaries }}=0\right.$ $\left.\mathrm{mol} / \mathrm{m}^{3}\right)$. Different values of the concentration gradient between the villi and capillary surfaces were tested.

To the best of the authors' knowledge, the diffusion coefficient of oxygen in placental tissue has not been directly measured. Some investigators have assumed the diffusion coefficient of the villous membrane to be similar to the oxygen diffusivity in plasma [2] and others to the diffusion coefficient of oxygen in water [15]. Due to the lack of data, a parametric evaluation of the diffusion coefficient was also performed.

There was no account for oxygen uptake by the villous stroma, nor the local pressure variations and other forces due to the maternal and fetal circulations. Stationary oxygen diffusion was assumed since the time for maternal blood to travel through the intervillous space (10-20 s) is considerably longer than the time for oxygen to diffuse into a villus (order of seconds) [1]. The solver run time ranged between 40 and 250 seconds.

Transport efficiency of the terminal villi has been suggested as the ratio between oxygen flux (Eq. 2) and capillary cross sectional area [2]. However, the area across which oxygen reaches the fetal bloodstream from the villous membrane is the surface area rather than the cross-sectional area of the capillaries. Therefore, a new oxygen transport efficiency which allows for geometric variability to be taken into account is proposed (Eq. 3).

$$
\begin{gathered}
J=-D \nabla \mathbf{C} \\
E=\frac{J}{V_{c a p}}
\end{gathered}
$$

The flux magnitude refers to the volumetric integration of the diffusive flux magnitude over the villous membrane, representing the amount of oxygen that will be absorbed by the capillaries and $V_{\text {cap }}$ refers to the capillary volume.

\section{Results}

\subsection{Geometrical Reconstructions}

Figure 2 shows the reconstructed geometries of the four specimens from the CLSM stacks, together with cross sectional slices for better visualization. Examples of the vasculo-syncytial 
Table 1: Geometrical details of the villi and capillary beds.

\begin{tabular}{|c|c|c|c|c|}
\hline Parameter & Specimen 1 & Specimen 2 & Specimen 3 & Specimen 4 \\
\hline Fixation Pressure [mmHg] & 100 & 100 & 30 & 30 \\
\hline Villous Volume $^{a}\left[\mu \mathrm{m}^{3}\right]$ & $2.59 \cdot 10^{6}$ & $2.25 \cdot 10^{6}$ & $3.06 \cdot 10^{6}$ & $3.21 \cdot 10^{6}$ \\
\hline Capillary Volume $\left[\mu \mathrm{m}^{3}\right]$ & $7.27 \cdot 10^{5}$ & $5.58 \cdot 10^{5}$ & $6.40 \cdot 10^{5}$ & $6.79 \cdot 10^{5}$ \\
\hline Volume Fraction ${ }^{b}[\%]$ & 28 & 25 & 21 & 21 \\
\hline Villous Surface Area $\left[\mu \mathrm{m}^{2}\right]$ & $1.23 \cdot 10^{5}$ & $1.28 \cdot 10^{5}$ & $1.59 \cdot 10^{5}$ & $1.35 \cdot 10^{5}$ \\
\hline Capillary Surface Area $\left[\mu \mathrm{m}^{2}\right]$ & $1.29 \cdot 10^{5}$ & $1.05 \cdot 10^{5}$ & $1.24 \cdot 10^{5}$ & $1.21 \cdot 10^{5}$ \\
\hline Surface Fraction $^{c}[\%]$ & 105 & 82 & 78 & 89 \\
\hline
\end{tabular}

membranes, usually defined as localized areas where the membrane separating the maternal and fetal blood streams are particularly thin [30], are indicated with arrows. It can also be observed that the capillaries have variable diameters, follow a sinuous course and form different types of loops.

Parameters of interest are directly obtained from the reconstructions and summarized in Table 1. Smaller values of the perfusion pressure resulted in a decrease of the volume ratio, defined as the ratio between the capillary volume to that of the villus. The surface area ratio (Eq. 4) between the capillaries and the villous membrane was 1.05, 0.82, 0.78 and 0.89 for specimen 1-4 respectively.

$$
A_{\text {ratio }}=\frac{\text { Capillary Surface Area }}{\text { Villi Surface Area }}
$$

\subsection{Diffusion Analyses}

An example of the distributions of the concentration and oxygen flux are provided in Figure 3 for each specimen. The vasculo-syncytial membranes, as expected, showed larger fluxes when compared to thicker areas of the tissue due to the small diffusion distances.

Parametric studies of the oxygen concentrations in the membrane and capillary surfaces showed no difference in the results when the driving oxygen gradient remained constant. However, the increase of the gradient had a profound effect in both the total oxygen flux and concentration magnitudes. Interestingly, the capillary boundaries that faced towards the center of the villi (opposite to the villous membrane) received very little oxygen (see Figure $3)$.

The oxygen flux magnitude was averaged (see Appendix A for details on local averaging) along different membrane thicknesses and was found to be inversely proportional to the villous membrane thickness (Figure 4). It can be recognized that, as expected, the maximum oxygen flux occurs at the thinnest barriers and the minimum at the thicker areas. The 

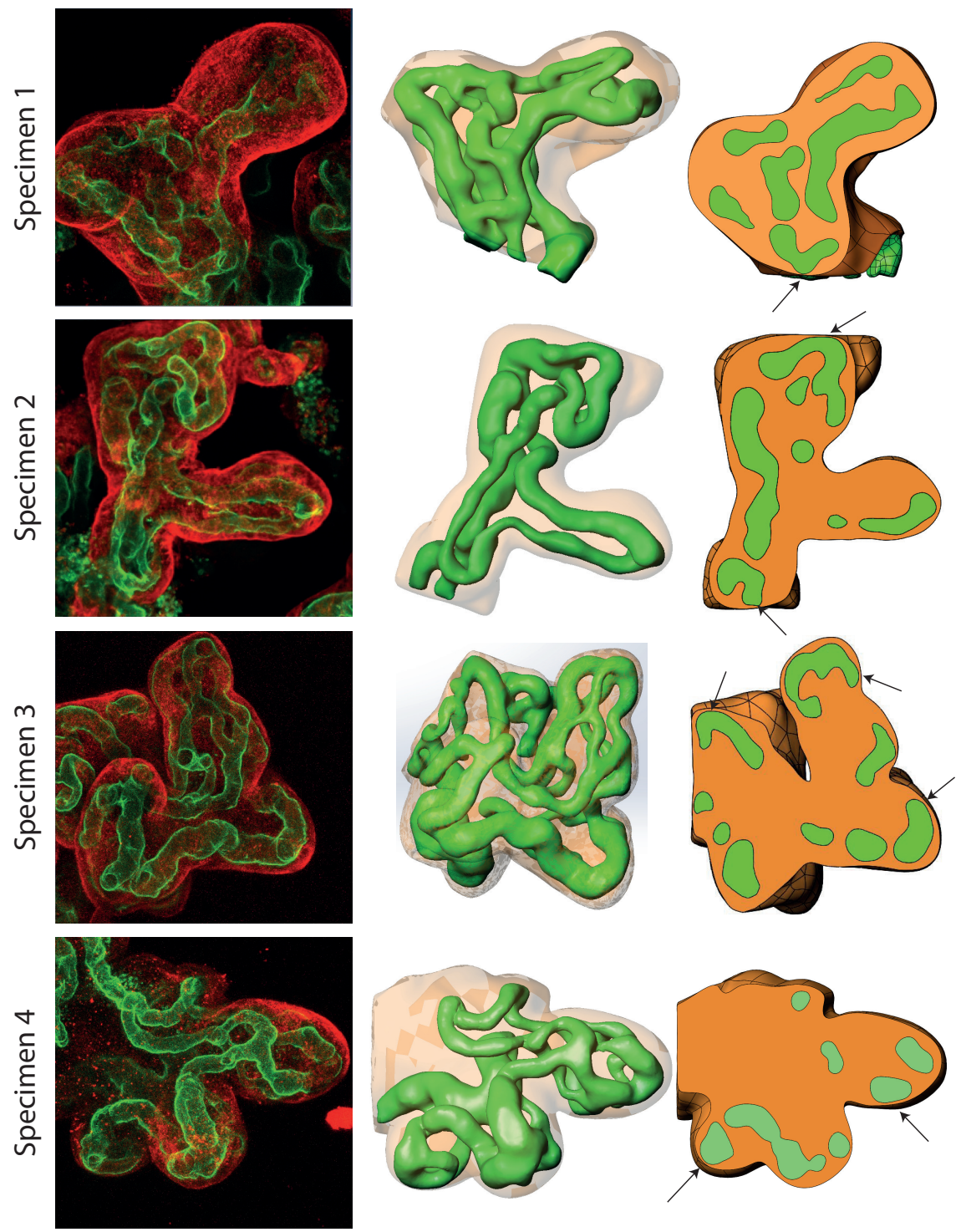

(a)

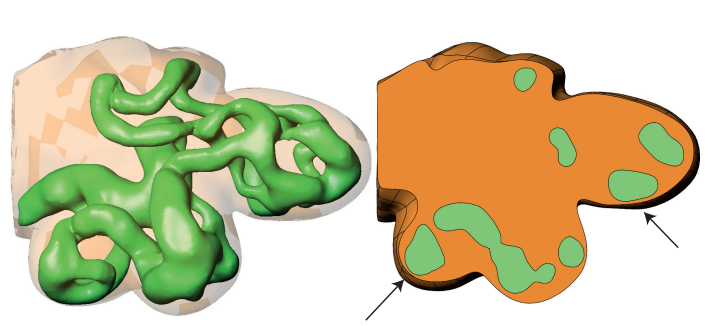

(b)

(c)

Figure 2: Geometrical reconstructions of the terminal villi and its capillary bed for all specimens. (a) 2D CLSM stacks, (b) Three-dimensional reconstructions and (c) Cut view showing different locations of vasculo-syncytial membranes. 

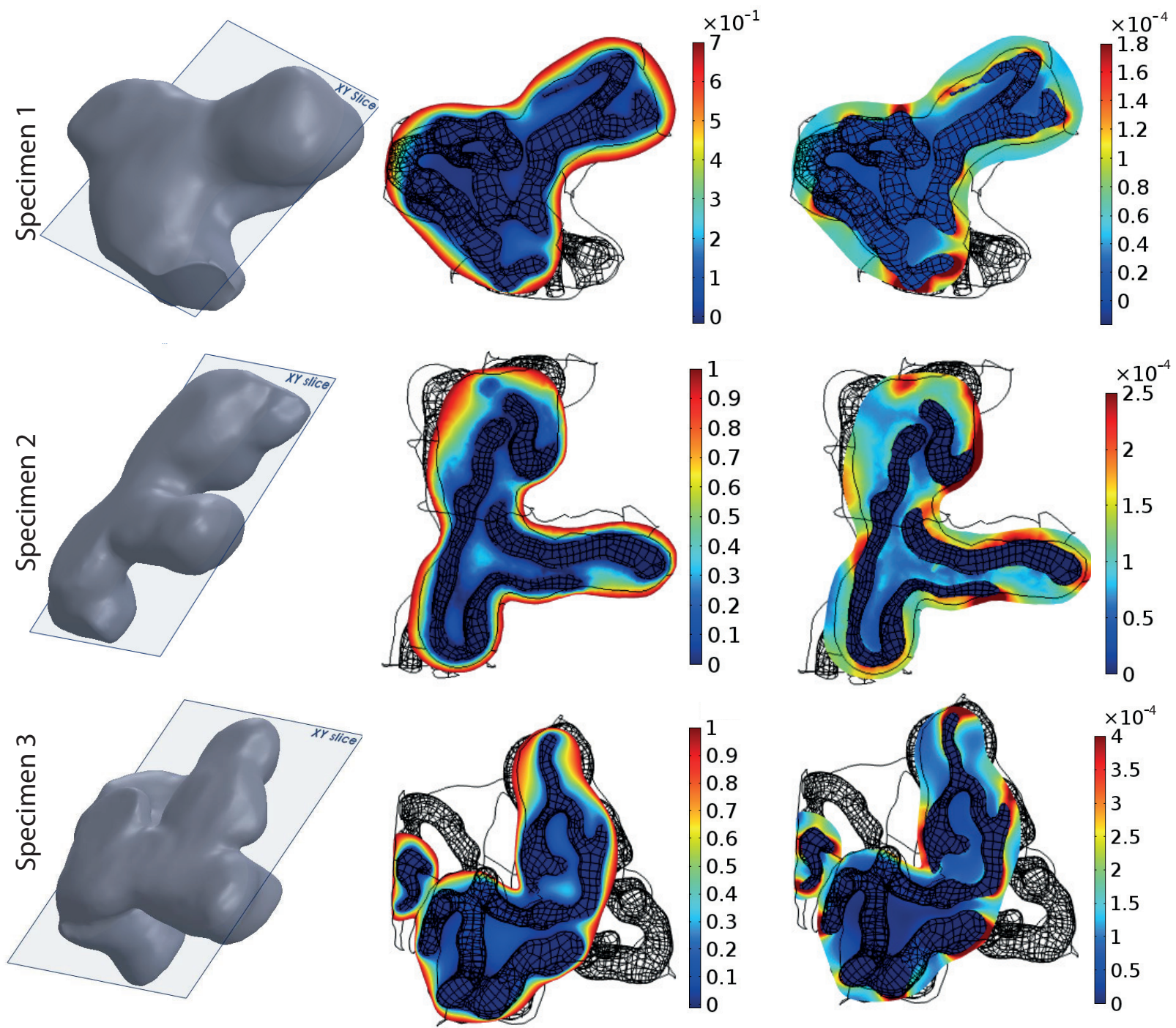

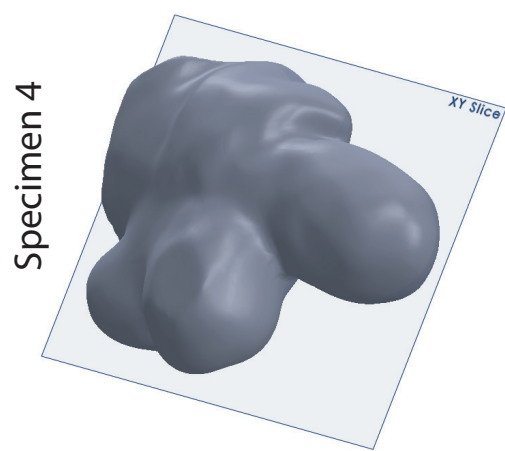

(a)

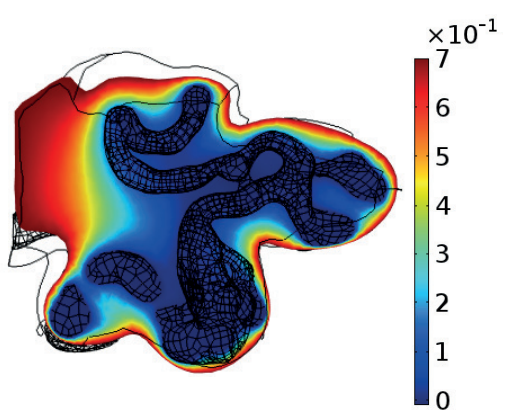

(b)

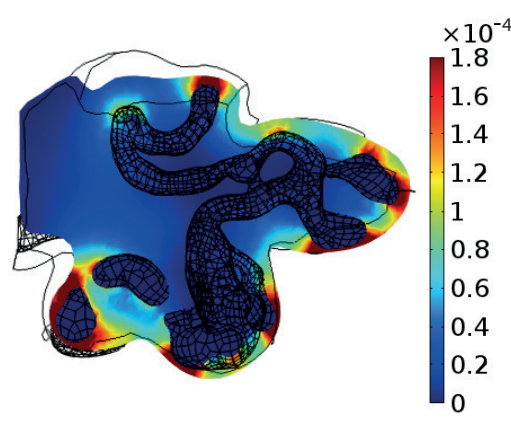

(c)

Figure 3: Distribution maps in a model with $D=1.62 \cdot 10^{-9} \mathrm{~m}^{2} / \mathrm{s}$. (a) Representative $x y$ slice, (b) Concentration $\left[\mathrm{mol} / \mathrm{m}^{3}\right]$ and (c) Flux magnitude $\left[\mathrm{mol} / \mathrm{m}^{2} \cdot \mathrm{s}\right]$ distributions. 


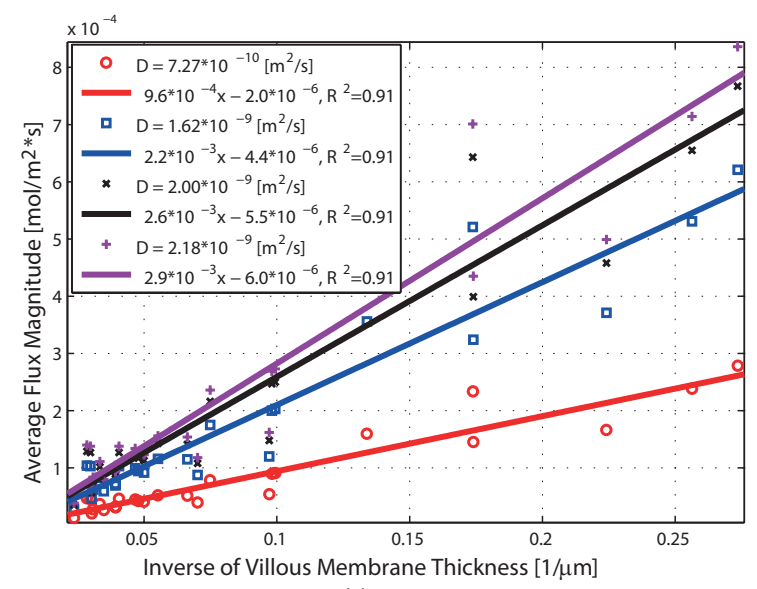

(a)

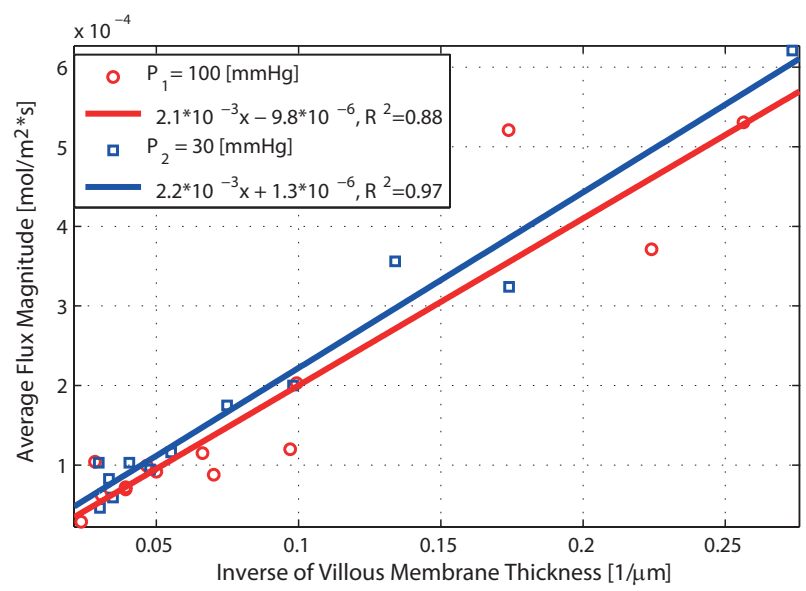

Figure 4: Average oxygen flux magnitude $\left[\mathrm{mol} / \mathrm{m}^{2} \cdot \mathrm{s}\right]$ vs. villous membrane thickness $[\mu \mathrm{m}]$. (a) Results of the parametric study for the diffusion coefficient, $D$, and (b) Comparison between the different fixation pressures, $P$.

diffusion coefficient has a significant effect on the rate and amount of oxygen that diffuses especially in the smallest distances.

The efficiency E, Eq. 3, of the terminal villi was $1.54 \cdot 10^{-4}, 2.89 \cdot 10^{-4}, 3.20 \cdot 10^{-4}$ and $1.90 \cdot 10^{-4}$ for specimens $1-4$ respectively (with $\mathrm{C}_{\text {villi }}=1 \cdot 10^{-9} \mathrm{~mol} / \mathrm{m}^{3}$ ).

\section{Discussion}

Research on the human placenta is challenging, mainly due to ethical considerations, the inaccessibility of the living organ, and the manipulation of the ex vivo tissue. Furthermore, there is considerable interspecies variation [31] which complicates the interpretation of animal studies. On the other hand, mathematical models are limited by the geometrical complexity of the system and its solute patterns $[2,20,32]$. As a consequence, the effect of certain parameters on the transport capacity and efficiency of the human placenta have not been effectively studied, leading to an urgent need for new research techniques. The current study was designed to assess the capabilities of specimen-specific FE analysis as an investigative tool for the human terminal villi.

Three-dimensional reconstructions showed different types of capillary loops and nonuniform capillary diameters, a feature that has been previously reported and referred to as sinusoids [30, 33]. It has been previously suggested that mechanical forces, e. g. pressure gradients, are responsible for sculpting these capillaries [1]. However, further research is still needed to clarify this idea and its effect on the fetal blood flow and diffusion capacity of the human placenta.

Even though there is a significant amount of data in the literature regarding the geometrical parameters of the villous components, most of them were calculated in a whole placenta rather than in a single terminal villus [3-5, 34-37]. Few works have calculated geometrical parameters such as volume fractions or surface area fractions in the terminal villus $[24,38,39]$; 
in agreement with Karimu et al. [39], averaged capillary to villi volume and area fractions were found to decrease with the fixation pressure. Both fractions were at least half of those reported by Karimu et al. [39] but in agreement with the lower values reported by Burton et al. [24]. The exact source of this discrepancy is unknown, but the different techniques for the estimation of parameters and the small sample cohort are believed to be related. Interestingly, some studies found the surface area of the villi to be bigger than that of the capillaries [17, 36, 40,41], while others reported the opposite [3, 5, 24, 42]; in this work both cases were found. Terminal villi geometries are variable within a single placenta and between villi [31] and therefore it is no surprise to find the different cases to be reported. The surface area ratio may be related to the amount of dilation of specific segments rather than to external factors since.

Most existing works used a morphometric approach to model 2D oxygen transport, where structural quantities were estimated by sectioning fixed sampled tissue and combined with physicochemical data. Many different aspects and scenarios of placental exchange have been studied using this technique $[3,5,15-18,43,44]$. However, 2D geometries cannot provide a full quantitative analysis of a 3D system because of inevitable geometrical and mathematical simplifications. A different approach is to simplify the placental geometry to allow for a numerical solution of a more complex scenario [21, 22]; a study done by Serov et al. [20] modeled a complex scenario (including maternal and fetal bloodstreams, membrane permeability and oxygen-hemoglobin dissociation curve) but simplified the terminal villi geometry to parallel tubes with constant diameters. This geometrical representation disables any function - structure relation, meaning that the different blood flow patterns (concurrent, counter-current or any other organization) provide the same results. To the best of the authors' knowledge this is the only work that applies FE analysis to the problem of oxygen transport across fully 3D terminal villi in the human placenta.

To obtain a reliable model, the geometry and the oxygen transport problem should describe as closely as possible the in vivo state. Here, the focus was initially on an accurate 3D reconstruction of randomly selected human placental terminal villi while a simplified mathematical description of the oxygen transport problem is solved. In this way, the FE capabilities to provide fast and accurate results can be tested. Figure 4 summarizes the importance of the membrane thickness on the total oxygen flux; not surprisingly larger diffusion coefficients led to higher oxygen transport fluxes. This behavior was predictable from Fick's law of diffusion where one can estimate the effect of the different $D$ on the flux, for a simplified model, to be $D \cdot \Delta C / h$ ( $h$ being the membrane thickness). Interestingly, the specimens which were perfused at the lower pressure (specimens 3 and 4) yielded larger values of oxygen flux in the thicker areas while those fixed at the higher pressure (specimens 1 and 2) transported larger amounts of oxygen in the thinner areas (Figure 4). It might be that the higher fixation pressure leads to more variability in the membrane thickness while the lower pressures resulted in a more even but thicker membrane. The surroundings of the vasculo-syncytial membranes appear to have an effect on the oxygen flux through them which does not only depend on a specific diffusion thickness. This effect can only be seen and investigated using a realistic 3D model of the terminal villi.

The concentration and flux distributions showed that while the vasculo-syncytial mem- 
branes transported large amounts of oxygen, the boundaries that faced towards the center of the specimen were exposed to little oxygen. This phenomenon supports the previously reported study on diffusional screening[2], whereby some capillary sections are shielded from receiving oxygen by others. An explanation could be that oxygen may diffuse out from the deeper capillary surfaces to supply the cells of the stromal core, but only a full computational model that includes the fetal bloodstream, the active transport and the trophoblast membrane oxygen consumption could clarify this phenomenon.

In the present study, the geometrical reconstructions largely depend on the staining quality of the tissues and the resolution of the CLSM. As a consequence, human interpretation is needed to remove imaging artifacts, which can sometimes be challenging. Currently, it is impossible to completely automate this methodology because manual cleaning is obligatory to define unclear boundaries and obtain smooth models. Additionally, the fetal blood pressure in the in vivo human placenta is estimated to be about $50 \mathrm{mmHg}$ [24], such that the fixation pressures may have represented the maximum and minimum fetal blood pressures. Therefore, the geometrical results may be overestimating or underestimating the real geometries. Another limitation is the number of terminal villi that can be analyzed simultaneously; a large number of villi such as in the work of Gill et al. [2] cannot be reconstructed altogether due to resolution problems. However, the presented methodology enables the different geometries to be deeply analyzed and the 3D geometry-function relation to be better understood with a more automatic approach and less user dependency than the one proposed by Gill et al. [2]. Finally, there are limitations associated with the difficulty to experimentally validate the results. Validations to support oxygen transport studies are challenging since the experimental conditions, such as the concentration of oxygen, culture medium and type of matrix, must be carefully adjusted [45].

Despite these limitations, CLSM 3D reconstructions together with FE models have the potential to become a valuable tool for placenta related research. The benefit of the proposed methodology is its application in simulation-based research aimed to serve as a basis for analyzing the transport capacity of human placentae. There is ongoing work to experimentally validate and improve the FE models to more complex but realistic scenarios where the fetal bloodstream and active transport are incorporated.

\section{Appendix A. Local Averaging}

Finite element analyses are advantageous because their outcome is in a detailed form, e.g. values on every point. Therefore, to extract results either on a line, surface or volume an averaging method is needed.

COMSOL Multiphysics automatically defines a line when selecting two points. For the diffusive flux magnitude reported in this study, random lines connecting the fetal capillary and the outer surface of the villous membrane representing the membrane thickness, were created. The flux was then averaged by a standard numerical integration method (quadrature) as follows: 


$$
\frac{\int_{a}^{b} f(x) d x}{b-a}
$$

where $f(x)$ if the flux at each point $x$ and a,b are the two extreme points of the line. This is the default averaging method of COMSOL Multiphysics which is implemented by the 'Line Averaging' command under the Results section.

\section{References}

[1] Burton GJ, Woods AW, Jauniaux E, Kingdom JCP. Rheological and Physiological Consequences of Conversion of the Maternal Spiral Arteries for Uteroplacental Blood Flow during Human Pregnancy. Placenta 2009;30(6):473-82.

[2] Gill JS, Salafia CM, Grebenkov D, Vvedensky DD. Modeling Oxygen Transport in Human Placental Terminal Villi. Journal of Theoretical Biology 2011;291(0):33-41.

[3] Ansari T, Fenlon S, Pasha S, O'Neill B, Gillan J, Green C, et al. Morphometric Assessment of the Oxygen Diffusion Conductance in Placentae from Pregnancies Complicated by Intra-Uterine Growth Restriction. Placenta 2003;24(6):618 -26.

[4] Mayhew TM, Sisley I. Quantitative Studies on the Villi, Trophoblast and Intervillous Pores of Placentae from Women with Well-controlled Diabetes Mellitus. Placenta 1998;19(5-6):371 -7.

[5] Mayhew TH, Joy CF, Haas JD. Structure-Function Correlation in the Human Placenta, the Morphometric Diffusing Capacity for Oxygen at Full. Journal of Anatomy 1984;139(4):691-708.

[6] Leiser R, Krebs C, Ebert B, Dantzer V. Placental Vascular Corrosion Cast Studies: A Comparison between Ruminants and Humans. Microsc Res Tech 1997;38:76-87.

[7] Burton G. The fine structure of the human placental villus as revealed by scanning electron microscopy. Scanning Microscopy 1987;1:1811-28.

[8] Langheinrich AC, Vorman S, Seidenstücker J, Kampschulte M, Bohle RM, Wienhard J, et al. Quantitative 3D Micro-CT Imaging of the Human Feto-placental Vasculature in Intrauterine Growth Restriction. Placenta 2008;29(11):937 -41.

[9] Langheinrich AC, Wienhard J, Vormann S, Hau B, Bohle RM, Zygmunt M. Analysis of the Fetal Placental Vascular Tree by X-ray Micro-Computed Tomography. Placenta 2004;25(1):95 - 100.

[10] Lisman BAM, van den Hoff MJB, Boer K, Bleker OP, van Groningen K, Exalto N. The Architecture of First Trimester Chorionic Villous Vascularization: A Confocal Laser Scanning Microscopical Study. Human Reproduction 2007;22(8):2254-60.

[11] Jirkovská M. Spatial Arrangement of the Microvascular Bed in the Human Placenta. Androl Gynecol: Curr Res 2013;1:4.

[12] Resta L, Capobianco C, Marzullo A, Piscitelli D, Sanguedolce F, Schena FP, et al. Confocal Laser Scanning Microscope Study of Terminal Villi Vessels in Normal Term and Pre-eclamptic Placentas. Placenta 2006;27(6-7):735 -9.

[13] Kirschbaym T, Shapiro N. A Mathematical Model of Placental Oxygen Transfer. Journal of Theoretical Biology 1969;25:380-402.

[14] Weibel ER. Morphometric Estimation of Pulmonary Diffusion Capacity: I. Model and Method. Respiration Physiology 1971;11(1):54 - 75.

[15] Bacon BJ, Gilbert RD, Kaufmann P, Smith A, Trevino FT, Longo LD. Placental Anatomy and Diffusing Capacity in Guinea Pigs following long-term Maternal Hypoxia. Placenta 1984;5:475-88.

[16] Longo L, Ching K. Placental Diffusing Capacity for Carbon Monoxide and Oxygen in Unanesthetized Sheep. Journal of Applied Physiology 1977;43:885-93.

[17] Mayhew TM, Jackson MR, Haas JD. Microscopical Morphology of the Human Placenta and Its Effects on Oxygen Diffusion: a Morphometric Model. Placenta 1986;7:121-31. 
[18] Reshetnikova OS, Burton GJ, Teleshova OV. Placental Histomorphometry and Morphometric Diffusing Capacity of the Villous Membrane in Pregnancies Complicated by Maternal Iron-deficiency Anemia. American Journal of Obstetrics and Gynecology 1995;173(3):724-7.

[19] Chernyavsky IL, Leach L, Dryden IL, Jensen OE. Transport in the Placenta: Homogenizing Haemodynamics in a Disordered Medium. Phil Trans R Soc A 2011;369(4162-4182).

[20] Serov AS, Salafia C, Brownbill P, Grebenkov DS, Filoche M. Optimal Villi Density for Maximal Oxygen Uptake in the Human Placenta. Journal of Theoretical Biology 2015;368:133-44.

[21] Costa A, Costantino ML, Fumero R. Oxygen Exchange Mechanisms in the Human Placenta: Mathematical Modelling and Simulation. Journal of Biomedical Engineering 1992;14:385-9.

[22] Gordon Z, Eytan O, Jaffa A, Elad D. Fetal Blood Flow in Branching Models of the Chorionic Arterial Vasculature. Annals of the New York Academy of Sciences 2007;:250-65.

[23] Serov AS, Salafia C, Grebenkov DS, Filoche M. The Role of Morphology in Mathematical Models of Placental Gas Exchange. J Appl Physiol 2016;120:17-28.

[24] Burton GJ, Ingram SC, Palmer ME. The Influence of Mode of Fixation on Morphometrical Data Derived from Terminal Villi in the Human Placenta at Term: A Comparison of Immersion and Perfusion Fixation. Placenta 1987;8:37-51.

[25] Karimu AL, Burton GJ. Significance of changes in fetal perfusion pressure to factors controlling angiogenesis in the human term placenta. Journal of Reproduction and Fertility 1994;102(2):447-50.

[26] Fedorov A, Beichel R, Kalpathy-Cramer J, Finet J, Fillion-Robin JC, Pujol S, et al. 3D Slicer as an Image Computing Platform for the Quantitative Imaging Network. Magnetic Resonance Imaging 2012;30(9):1323-41.

[27] Shapiro LG, Stockman GC. Computer Vision. Prentice Hall; 2002.

[28] Lorensen WE, Cline HE. Marching Cubes: A High Resolution 3D Surface Construction Algorithm. In: Proceedings of the 14th Annual Conference on Computer Graphics and Interactive Techniques. 1987, p. $163-9$.

[29] Szabo B, Babuska I. Introduction to Finite Element Analysis: Formulation, Verification and Validation. Wiley-Blackwell; 2011.

[30] Burton GJ, Tham SW. The Formation of Vasculo-Syncytial Membranes in the Human Placenta. Journal of Developmental Physiology 1992;43-47.

[31] Benirschke K, Burton GJ, Baergen RN. Pathology of the Human Placenta. Springer; 2012.

[32] Chernyavsky IL, Jensen OE, Leach L. A Mathematical Model of Intervillous Blood Flow in the Human Placentone. Placenta 2010;31(1):44 - 52.

[33] Jirkovská M, Kubínová L, Janáček J, Kaláb J. 3D Study of Vessels in Peripheral Placental Villi. Image Analysis \& Stereology 2007;26(3):165-8.

[34] Rainey A, Mayhew T. Volumes and Numbers of Intervillous Pores and Villous Domains in Placentas Associated with Intrauterine Growth Restriction and/or Pre-eclampsia. Placenta 2010;31(7):602 -6.

[35] Mayhew TM. A Stereological Perspective on Placental Morphology in Normal and Complicated Pregnancies. Journal of Anatomy 2009;215:77 - 90.

[36] Mayhew TM, Manwani R, Ohadike C, Wijesekara J, Baker PN. The Placenta in Pre-eclampsia and Intrauterine Growth Restriction: Studies on Exchange Surface Areas, Diffusion Distances and Villous Membrane Diffusive Conductances. Placenta 2007;28(2-3):233 -8.

[37] Egbor M, Ansari T, Morris N, Green C, Sibbons P. Pre-eclampsia and Fetal Growth Restriction: How Morphometrically Different is the Placenta? Placenta 2006;27(6-7):727 -34.

[38] Burton GJ, Reshetnikova OS, Milovanov AP, Teleshova OV. Stereological Evaluation of Vascular Adaptations in Human Placental Villi to Differing Forms of Hypoxic Stress. Placenta 1996;17(1):49 55.

[39] Karimu A, Burton G. The Effects of Maternal Vascular Pressure on the Dimensions of the Placental Capillaries. British Journal of Obstetrics and Gynaecology 1994;101:57-63.

[40] Egbor M, Ansari T, Morris N, Green CJ. Morphometric Placental Villous and Vascular Abnormalities in Early- and Late-onset Pre-eclampsia with and without Fetal Growth Restriction. BJOG : An International Journal of Obstetrics and Gynaecology 2006;113(5):580-9. 
[41] Mayhew TM, Wijesekara J, Baker PN, Ong SS. Morphometric Evidence that Villous Development and Fetoplacental Angiogenesis are Compromised by Intrauterine Growth Restriction but not by Pre-eclampsia. Placenta 2004;25(10):829 -33.

[42] Burton GJ, Jauniaux E. Sonographic, Stereological and Doppler Flow Velocimetric Assessments of Placental Maturity. British Journal of Obstetrics and Gynaecology 1995;102:818-25.

[43] Mayhew TM. Estimating Oxygen Diffusive Conductances of Gas-Exchange Systems: A Stereological Approach Illustrated with the Human Placenta. Annals of Anatomy 2013;

[44] Reshetnikova OS, Burton GJ, Milovanov AP. Effects of Hypobaric Hypoxia on the Fetoplacental Unit: The Morphometric Diffusing Capacity of the Villous Membrane at High Altitude. American Journal of Obstetrics and Gynecology 1994;171(6):1560 -5.

[45] Schneider H. Oxygenation of the Placental-Fetal Unit in Humans. Respiratory Physiology \& Neurobiology 2011;178(1):51-8. 